\title{
Avons-nous besoin des EMH? De quoi les EMH ont-elles besoin?
}

\section{Ruedi Bienz}

A l'occasion de son départ en tant que directeur des EMH

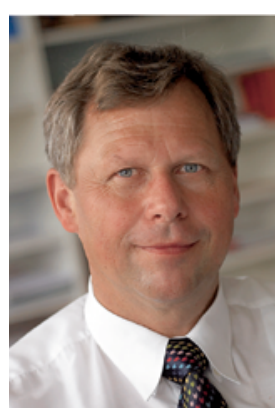

Lors d'un départ, on entend souvent parler d'«un enfant qu'on laisse entre de bonnes mains» ou de «l'engagement passionné avec lequel pendant des années...». Je préfère rester un peu plus sobre et jeter un coup d'œil au passé, mais surtout à l'avenir.

En 1997, un groupe de travail de la FMH constitué de son président de l'époque, Hans Heinrich Brunner, du membre du comité central Ludwig Heuss et du rédacteur en chef du BMS Reto Steiner a recherché le dialogue avec différentes maisons d'édition suisses. L'objectif visé était de travailler avec une maison d'édition possédant l'expertise médicale et éditoriale nécessaire pour atteindre les objectifs suivants avec la FMH:

- façonner le journalisme médical en Suisse à travers des publications de haute qualité, indépendantes au niveau rédactionnel;

- renforcer la FMH et la cohésion entre ses membres;

- donner une image positive du corps médical au public.

La procédure d'évaluation aboutit à la collaboration avec les éditions scientifiques Schwabe, dont le concept avait convaincu et qui avaient pu apporter leurs propres revues et ouvrages médicaux à la maison d'édition commune. Les Editions Médicales Suisses EMH qui détail non négligeable - devaient également permettre aux partenaires concernés de réaliser des bénéfices, ont été fondées le 18 septembre 1997.

Il est vite apparu que les objectifs ambitieux étaient très idéalistes par rapport à la réalité du journalisme médical en Suisse. Le nœud du problème est resté le même jusqu'à aujourd'hui: la grande force des produits EMH est aussi leur talon d'Achille. Toutes les revues publiées par les EMH sont totalement indépendantes au niveau rédactionnel et exclusivement axées sur les attentes des lecteurs. Cette approche sans concession est à l'origine de la confiance que ces derniers accordent à nos produits. Dans les sondages de lecteurs indépendants [1, 2], les revues EMH arrivent toujours en tête, loin devant la concurrence. Le concept de séparation stricte de la rédaction et du marketing est plus problématique lorsqu'il s'agit d'attirer des fonds publicitaires de l'industrie, les annonceurs obéissant à d'autres critères.

\footnotetext{
Les résultats de «L'enspécialisée 2017» sont www.emh.ch/fr/emh/ enquete-lecteurs.htm Les résultats de «'en quête presse médicale www.emh.ch/fr/info/ En 2018, chaqu membre de la FMH des verse plus qu'une clés des $\mathrm{EMH}$ A partir

de base est entièrement supprimée.
}

r.bienz[at]emh.ch
Les difficultés financières résultant de cette constellation initialement sous-estimée ont conduit à ma nomination au poste de directeur des EMH par les deux actionnaires FMH et Schwabe au tournant du millénaire. Avec le soutien des actionnaires et grâce à une équipe motivée, il a été possible de mener les EMH au succès. 21 ans après leur création, les EMH existent toujours et jouissent d'une bonne santé financière. Mais les défis auxquels elles sont confrontées dans un paysage médiatique qui change à toute vitesse sont considérables.

En tant que directeur sortant, je suis libre de parler ou plutôt d'écrire sans détour. Pour perpétuer le modèle des $\mathrm{EMH}$, il faut que le corps médical, que vous, chères lectrices et chers lecteurs, souteniez les EMH dans leur mission telle que décrite ci-dessus. Il me semble crucial que vous soyez conscients de l'étendue de cette mission, de l'importance d'une maison d'édition propre et de la responsabilité qu'elle représente et que vous partagiez cette dernière. Chaque semaine, les EMH vous font parvenir (presque) gratuitement [3] le Bulletin des médecins suisses et le Swiss Medical Forum. Avec le Swiss Medical Weekly, les EMH vous proposent également une plateforme de publication scientifique au facteur d'impact considérable. Enfin, les EMH offrent aux sociétés de discipline médicale une infrastructure professionnelle pour une gestion moderne et peu coûteuse de leur communication. Ces services ne sont pas financés par votre cotisation, mais par des fonds publicitaires durement gagnés.

Pour pouvoir continuer à exister dans un contexte médiatique aux mutations rapides et à la concurrence intense, les EMH ont besoin de voies décisionnelles courtes, de souplesse et de liberté d'entreprendre. C'est pourquoi je conclus sur ce souhait et cette prière que je vous adresse, chères lectrices et chers lecteurs, chers membres de la FMH: accordez la liberté nécessaire aux EMH par le biais des instances compétentes de la FMH, faites confiance au conseil d'administration et permettez aux EMH de faire du bon travail.

.

